\title{
COMPARAISON DES LANGUES ET GRAMMAIRE COMPARÉE. RÉFLEXIONS MÉTHODOLOGIQUES.
}

Dans cet article je voudrais aborder un problème historiographique et méthodologique: le passage de la comparaison de langues à la grammaire comparée. Ce passage reflète une certaine continuité - au niveau des matériaux et de l'analyse comparative d'un certain nombre de langues - mais il témoigne d'une discontinuité radicale que les histơriens de la linguistique interprètent souvent comme un processus de sophistication allant vers la création d'un paradigme "scientifique". C'est aussi le sentiment de ceux qui font partie des gẻnérations "paradigmatiques". Ainsi, Antoine Meillet $\mathbf{n}$ 'hésite pas à dater l'émergence de la grammaire comparée: "La grammaire comparée a été créée au début du XIXe siècle par les savants allemands et danois"'. On aurait mauvaise grâce à soupçonner le maître français de myopie historique: Meillet est conscient de la longue préhistoire du comparatisme, mais il prend soin de la distinguer de la grammaire comparée. La pierre de touche ici est la méthode, et il n'est donc pas étonnant que le premier chapitre de l'Introduction de Meillet porte sur la méthode de la grammaire comparée. La grammaire comparée, dont l'objet est l'étude de concordances que présentent certaines langues entre elles, s'insère dans le contexte des "recherches méthodiques que le XIXe siècle a instituées sur le développement historique des faits naturels et sociaux “2. Or, qu'est-ce qui permet de définir la "grammaire comparée“? Je distinguerais trois composantes (qui sont intimement liées) dans la grammaire comparée:

1. la notion de "forme grammaticale"

2. la notion de "type linguistique"

3. la notion de "changements systématiques" en tant que transitions.

Dans la suite, je développerai chacune de ces trois composantes, en indiquant leur portée, leur interdépendance, et leur maturation historique. Chacune de ces composantes correspond à des aspects du problème général de la comparaison des langues, et de la justification même de leur description comparative.

La notion de forme grammaticale correspond au problème du paramètre de comparaison: que faut-il (que peut-on) comparer, quand on juxtapose des langues?

\footnotetext{
A. Meillet, Introduction à l'étude comparative des langues indo-européennes, Paris, Hachette, $1937^{8}$ (19031), p. 453; cf. P. Swiggers, "La linguistique historico-comparative d'Antoine Meillet: Théorie et méthode". Cahiers Ferdinand de Saussure 39, 1985, pp. 181-195 (183-184).

2 A. Meillet, o.c., p. 456; sur la méthode comparative comme concept historiographique, voir H. Hoenigswald, "On the history of the comparative method", Anthropological Linguistics 5, 1963, pp. 1-11; T. Orlandi, "La metodologia di Franz Bopp e la linguistica precedente", Rendiconti dell'Istituto Lombardo di Scienze e Lettere, Classe di Lettere e scienze morali e storiche 96, 1962, pp. 529-549; R. Wells, "Linguistics as a science: the case of the comparative method", dans H. Hoenigswald éd., The European Background of American Linguistics, Dordrecht, Reidel, 1979, p. 23-61.
} 
Le comparatisme du XVIe, du XVIIe et du XVIIIe siècle, tout en reconnaissant la possibilité d'une segmentation plus fine - d'où des remarques sur les correspondances entre des "lettres" —, s'en tient à la comparaison de mots (ou de lexèmes constitués): numéraux (voir déjà chez Sassetti, B. Schulze, Coeurdoux) ${ }^{3}$, noms divins, noms de parenté. Les résultats sont intéressants, non seulement en ce qu'ils révèlent une profonde similitude - que celle-ci soit interprétée comme une preuve de parenté génétique ou comme le témoignage de contacts intensifs, a moins d'importance ici - , mais aussi parce qu'ils portent parfois sur des unités lexématiques qui entrent parfois dans des mots plus complexes: je pense ici aux prépositions/préfixes ${ }^{4}$. La grammaire comparée approfondira l'analyse en dégageant des formes grammaticales, constituées d'un radical et d'affixes (préfixes, infixes, suffixes) - plus tard viendra la "découverte" des formatifs thématiques, et la distinction entre suffixes dérivationnels et suffixes flexionnels. Peu importe ce raffinement théorique à l'intérieur de la grammaire comparée - on confrontera sur ce point l'introduction de la Vergleichende Grammatik de Bopp à celle du Compendium de Schleicher ${ }^{5}$, et au chapitre "Principes de la morphologie" dans l'Introduction de Meillet (on remarquera à ce propos (1) l'extension progressive du traitement, et (2) son déplacement, de matière liminale à charpente). Peu importe aussi l'avance que manifeste le "comparatisme sémitique à l'égard de la grammaire indoeuropéenne: tirant profit de la structure des langues sémitiques, les lexicographes arabes du Moyen Age étaient déjà arrivés à traiter séparément radicaux et affixes et à décrire même les consonnes par rapport à leur rôle dans les deux types de seg-

3 Pour Sassetti; voir surtout sa lettre à Bernardo Davanzati (lettre du 22 janvier 1586): "Sono scritte le loro scienze tutte in una lingua, che dimandono sanscruta, che vuol dire bene articolata, della quale non si ha memoria quando fusse parlata, con avere (com'io dico) memorie antichissime. In parlarla come noi la greca e la latina vi pongono molto maggior tempo, si che in sei anni o sette se ne fanno padroni: e ha la lingua d'oggi molte cose comuni con quella, nella quale sono molti de'. nostri nomi, e particularmente de' numeri 6, 7, 8, e 9, Dio, serpe, e altri assai“ (cf. I. -C. Muller, Early stages of language comparison from Sassetti to Sir William Jones (1786)", Kratvlos 31, 1986, pp. 1-31, ici p. 15). De Schulze, voir sa lettre à Th. A. Francke (lettre du 23 août 1725): "Als ich kurz darauf.Kirendum anfing zu lernen, so befand ich, dass sie in ihrcr Numeration fast lauter pure lateinisçhe Wörter hätten. Hier fragt sichs: Woher die Brahmanen diese Wörter gekriegt? Ob sie selbige von der Portugiesischen Sprache abgeborgt, die nun mehro 200 lahr in Indien bekannt worden. oder ob sie selbige vor vielen Jahren her von den Römern und alten I ateinern bekommen?" (cf. D. Droixhe, La linguistique et l'appel de l'histoire. Rationalisme et révolutions positivistes, Genève. Droz. 1978, pp. 76-77; J.-C. Muller, "Quelques repères pour l'histoire de la notion de vocabulaire de hase dans le précomparatisme", Histoire, Epistémologie, Langage 6:2, 1984, pp. 37-43, ici p. 38).

Sur la contribution de Coeurdoux, voir M. Mayrhofer, Sanskrit und die Sprachen Alteuropas. Zwei Jahrhunderte des Widerspiels von Entdeckungen und Irrtümern, Göttingen (Nachrichten der Akademie der Wissenschaften in Göttingen, Phil.-Hist. Klasse, Jg. 83: 5), 1983, et J.-C. Muller, "Early stages..." a.c., pp. 24-25.

4 Comme c'est le cas chez A. Schrieckius (Van t'beghin der eerster volcken van Europen, in-sonderhevt vanden oorspronck ende saecken der Neder-landren, XXIII boecken. Ypre, F. Bellet, 1614); cf. P. Swiggers "Adrianus Schrieckius: de la langue des Scvthes à l'Europe linguistique". Histoire. Epistémologie, Langage 6:2, pp: 17-34.

5 F. Bopp, Vergleichende Grammatik des Sanskrit, Send, Armenischen, Griechischen. Lateinischen. Litauischen, Altslavischen, Gothischen und Deutschen, Berlin, F. Dümmler, 186833; A. Schleicher, Compendium der vergleichenden Grammatik der indogermanischen Sprachen, Weimar, Böhlau, $1861-1862$ ( 2 volumes). 
ments $^{6}$. Ce qui importe, par contre, c'est l'emploi technique de la forme grammaticale au niveau de la comparaison. Ainsi, on voit que le comparatisme fait parfois appel à la "structure grammaticale interne" (ou la "compositio"): c'est le cas chez Thomas Stephens (1549-1619) - dans sa lettre du 24 octobre 1583 à son frère $\mathrm{Ri}$ chard $^{7}$ - , chez Saumaise ${ }^{8}$, et chez Leibniz, dans sa correspondance avec Ludolf et dans la Brevis designatio ${ }^{9}$. Mais qu'en résulte-t-il concrètement? En tout cas, pas de comparaison de structures grammaticales spécifiques. Et Leibniz accorde encore un poids décisif à la similitude observée entre des lexèmes. Qu'en est-il de Hümboldt? On sait que c'est chez lui qu'est explicitée la notion de "forme grammaticale" comme corrélation médiatrice entre une substance linguistique et "das Denken"10. Les formes grammaticales sont un mode particulier de représentation de rapports grammaticaux, et doivent être situées par rapport aux différents stades de la formation (progressive) de la grammaire. La forme grammaticale a un double rôle dans l'interaction de la pensée et de la langue: elle est à la fois un instrument "passif"; résultant d'une codification (par l'intermédiaire des catégories grammaticales) des expressions de la pensée, et un instrument actif, qui permet au locuteur d'exprimer ses idées avec plus de clarté. Dans l'expression des rapports qu'elles ont à exprimer, les langues diffèrent, "techniquement" " par l'élaboration des formes grammaticales: "Darum, dass sich mit den Bezeichnungen fast jeder Sprache alle grammatischen Verhältnisse andeuten lassen, besitzt noch nicht auch jede grammatische Formen in demjenigen Sinne, in dem sie die hochgebildeten Sprachen kennen: Der zwar feine, aber doch sehr fühlbare Unterschied liegt in dem materiellen Erzeugniss und der formalen Einwirkung “11.

La forme grammaticale met en rapport une certaine matière et une forme particulière. Mais c'est dans la mise en relation de ces deux aspects que les langues peuvent différer de façon significative: on aurait tort de considérer les formes de lan-

6 Cf. par exemple les classifications chez Aharon ben Moses ben Asher et chez. Saadia Gaon, commentées dans notre article "I. 'histoire de la grammaire hébrä̈que jusqu'au XVIe siècle“", Orientalia L.ovaniensia Periodica 10, 1979. pp. 183-193.

7 Le jésuite anglais Thomas Stephens (1549-1619) écrit, le 24 octobre 1583, à son frère une lettre à propos des langues de l'Inde: "I.ingue harum regionum sunt permultae. Pronunciationem habent non invenustam et compositione latinae grecaeque similem; phrases et constructiones plane mirabiles. 1 . iterae syllabarum vim habent, quae toties variantur quoties consonantes cum vocabulis, vel mutae cum liquidis combinari possunt".

\& Cl. Saumaise, De hellenistica commentarius, controversiam de lingua hellenistica decidens et plenissime pertractans originem ac dialectos graecae linguae, Leiden. Elsevier. 1643; cf. les analyses de D. Droixhe, o.c., pp. 90-91, et de J.-C. Muller. "Salimaise. Monboddo. Adelung: vers la grammaire comparée". dans S. Auroux et al. (éds). Matériaux pour une histoire des théories linguistiques. Lille. Presses universitaires, 1984, pp. 389-396.

9 Sur la contribution linguistique de I ceibniz. voir $\mathrm{H}$. Aarsleff, "The study and use of etymology in l.eibniz". Studia Leibnitiana - Supplementa III, 1969. pp. 173-189; S. von der Schulenberg, I.eihniz als Sprachforscher. Frankfurt. Klostermann, 1973; . . T. Waterman, I.eibniz and Ludolf on things linguistic. Excerpts from their correspondence (1.688-1703), Berkeley. University of California Press. 1978.

10 Cf. P. Swiggers. "Catégories grammaticales et catégories culturelles dans la philosophie du langage de Humholdt: les implications de la 'forme grammaticale" ". Teitschrift, für Phonetik. Sprachwissenschaft und Kommunikationsforschung 38. 1985. pp. 729-736.

11 W. von Humboldt. Werke in fïnf Bänden (éd. par A. Flitner et K. Giel), Darmstadt, Wissenschaftliche Buchgesellschaft, 1961-1964. Band III: Schriften sur Sprachphilosophie. p. 34. 
gues différentes comme étant grammaticalement équivalentes même s'il y a des correspondances dans la traduction. En quoi consiste donc la (vraie) forme grammaticale? Pour Humboldt, elle consiste en la fusion - ou l'intégration mutuelle — de la matière et de la forme. La matière provient de l'aperception de l'objet: elle est l'idée correspondant à la désignation des objets tels qu'ils sont appréhendés. À l'état pur, la matière est exprimée par des mots séparés, dont la fonction ne consiste qu'à nommer les objets. La forme correspond à un acte créatif, non appréhensif de l'objet; elle sert à établir le lien entre les éléments du discours: "Die Wörter, und ihre grammatischen Verhältnisse sind zwei in der Vorstellung durchaus verschiedne Dinge: Jene sind die eigentlichen Gegenstände in der Sprache, diese bloss die Verknüpfungen, aber die Rede ist nur durch beide zusammengenommen möglich. Die grammatischen Verhältnisse können, ohne selbst in der Sprache überall Zeichen zu haben, hinzugedacht werden, und der Bau der Sprache kann von der Art Seyn, dass Undeutlichkeit und Misverstand dabei dennoch, wenigstens bis auf einen gewissen Grad, vermieden werden" ${ }^{12}$.

Pour l'expression de la forme, on dispose de trois moyens:

(1) On peut susciter dans l'esprit l'idée d'un rapport - sans que celui-ci soit marqué linguistiquement - par l'assemblage des mots: dans ce cas, la langue possède une grammaire sans formes grammaticales proprement dites.

(2) On peut marquer le rapport par un signe qui le représente à la façon des objets eux-mêmes.

(3) On intègre l'expression des rapports à celle des objets, les rapports étant des "modificatifs" des objets. L'unité de cette forme grammaticale est souvent marquée par l'accent.

On aura reconnu ici les jalons du parcours typologique allant des langues isolantes aux langues flexionnelles. Cette typologie, trop peu nuancée, est opératoire en ce qu'elle permet d'apprécier globalement à quel stade formatif se trouve une langue au moment de l'observation. L'opposition qui se manifeste ici est celle entre langues "à grammaire plate" (ou grammaire lexicalisée) et celles à lexique grammaticalisé (tout mot y étant grammaticalement codé) ${ }^{13}$. Humboldt oppose ainsi la langue chinoise aux langues indo-européennes. Dans ces dernières, chaque mot appartient à une partie du discours déterminée, et n'a plus uniquement une individualité lexicale, mais également grammaticale. Dans la langue chinoise, les mots peignent les choses, représentées de façon discrète dans le discours; du discours, ils n'offrent que le contenu: "La langue chinoise emploie tous les mots dans l'état où ils indiquent l'idée qu'ils expriment, abstraction faite de tout rapport grammatical. Tous les mots chinois, quoique enchaînés dans une phrase, sont in statu absoluto, et ressemblent parlà aux radicaux de la langue samscrite (...). La langue chinoise renonce à la distinction précise et minutieuse des cátegories grammaticales, range les mots des phrases

\footnotetext{
W. von Humboldt, o.c., pp. 37-38.

13. Cf. P. Swiggers, "Forme et sens dans la linguistique du Siècle des Lumières", Beiträge zur romanischen Philologie 25, 1986, pp. 143-147.
} 
d'après l'ordre moins restreint de la détermination des idées, et donne aux périodes une structure à laquelle ce système est applicable. La langue samscrite, les langues qui ont une affinité évidente avec elle, et peut-être d'autres encore sur lesquelles je ne voudrais rien préjuger ici, établissent la distinction des catégories grammaticales comme base unique de leur grammaire, poursuivent cette distinction jusque dans leurs dernières ramifications, et s'abandonnent, dans la formation de leurs phrases, à tout l'essor que ce guide sûr et fidèle leur permet de prendre. La langue grecque surtout, jouit de cet avantage“14.

Pour ce qui concerne l'emploi qui est fait de cette notion de forme grammaticale, on constate que chez Humboldt elle sert surtout à opposer des types de langue, et non à justifier l'organisation interne de la grammaire comparée. Ce n'est qu'avec Bopp et plus tard avec Schleicher que la segmentation des formes grammaticales en indo-européen permettra

(1) de poser le problème de l'appartenance typologique (du point de vue de la morphologie) de l'indo-européen et des différentes langues indo-européennes historiquement attestées. Rappelons ici la postulation d'un stade d'agglutination primitif - postulation qui n'est pas sans rapport avec des modèles d'analyse syntaxique courants aux XVIIe et XVIIIe siècles (il suffit de penser à l'analyse chez Bopp du morphème dérivationnel sya du futur en indo-iranien comme une forme du verbe copule) - , et son évolution vers un type flexionnel à radical variable;

(2) de définir la famille indo-européenne à l'égard d'autres familles; c'est ce que fera Schleicher (Compendium) en séparant les langues indo-européennes des langues sémitiques, et des langues tartares ${ }^{15}$. Plus tard, la valeur de discrimination que possède la notion grammaticale sera exploitée par Trubetzkoy; Benveniste et Kurylowicz $^{16}$ : Humboldt est donc plutôt un représentant du comparatisme, que de la grammaire comparée. On le voit d'ailleurs dans sa lettre à August Wilhelm Schlegel que Henry Hoenigswald a analysée ${ }^{17}$ : la classification généalogique des langues doit avant tout être basée sur."der lexikalische Teil der Sprache" (vocabulaire de base + certains morphèmes liés). La grammaire comparée se développera en choisissant comme paramètre de comparaison des structures: morphologiques d'abord (Bopp); morphologiques et phonétiques. (Schleicher - s'appuyant sur les recherches étymologiques de Pott et Fick), et (morpho-)syntaxiques plus tard (travaux de Krahe, Kuryłowicz, Benveniste, Bader, Friedrich, Lehmann, Watkins, Schmalstieg, Justus).

J'en viens maintenant à la deuxième notion, celle de type linguistique. Cette notion correspond au domaine de la comparaison. Plus particulièrement, l'élaboration

14 W. von Humboldt. De l'origine des formes grammaticales, suivi de Lettre à $M$. Abel Rémusat (éd. par Ch. Porset), Bordeaux, Ducros, 1969, pp. 74, 108-109.

15 A. Schleicher, Compendium der vergleichenden Grammatik der indogermanischen Sprachen, Weimar; Böhlau, $1876^{4}$, pp. $2-4$ :

16. Cf. N. Trubetzkoy, "Gedanken ïher das Indogermanenproblem", Acta Linguistica 1, 1939, pp. 81-89; E. Benveniste, Origines de la formation des noms en indo-européen,' Paris, AdrienMaisonneuve, 1935; J. Kurytowicz, The Inflectional Categories of Indo-European, Heidelberg, C. Winter, 1964.

17 H. M: Hoenigswald, "Etymologv against grammar in the early 19th centurv", Histoire, Epistémologie, Langage 6, 1984, pp. 95-100. 
de la notion correspond à une nécessité pragmatique: celle de poser des principes contraignants qui, devant une gamme d'explications équivalentes, permettent de renforcer la probabilité de l'une d'entre elles. Prenons le cas de la comparaison des langues: l'objectif est d'expliquer des concordances (similitudes) observées entre des langues. Or, celles-ci se prêtent à 5 types d'explications: (1) les concordances résultent du hasard (et on connaît de tels cas); (2) les concordances résultent d'un emprunt (éventuellement à distance, ou par interposition); (3) les concordances sont le résultat d'une diffusion aréale; (4) les concordances résultent de l'unité foncière du langage (ce que Schuchardt appellera "Elementare Verwandtschaft") ${ }^{18}$; (5) les concordances reposent sur une unité phylogénétique, et les langues qui manifestent ces concordances remontent à un ancêtre commun. Notons que les réponses 1,2 et 5 sont les options dont on dispose en critique textuelle: innovations (ou fautes) communes (et indépendantes), leçons (éventuellement des fautes) copiées de l'autre témoin, leçons ou fautes communes provenant d'un ancêtre commun. Pour démontrer la parenté de langues présentant des concordances (ou pour la rendre très probable), la notion de type linguistique est cruciale: c'est à partir de la typologie qu'on peut définir des structures grammaticales, et - faut-il le rappeler encore après Meillet ${ }^{19}$ - que la grammaire comparée rapproche des séries entières manifestant des homologies structurelles (et sa.valeur probante est la plus grande, quand ces homologies structurelles concernent des séries de formes irrégulières). On comprend ainsi pourquoi F. Schlegel écrit: "Mais le point décisif qui éclairera tout, c'est la structure interne des langues ou la grammaire comparée, laquelle nous donnera des solutions toutes nouvelles sur la généalogie des langues, de la même manière que l'anatomie comparée a répandu un grand jour sur l'histoire naturelle" ${ }^{\text {"20 }}$. Reste que dans la projection de cette typologie sur l'axe de l'histoire, les comparatistes admettent le passage (ou la déchéance) d'un type à l'autre. La typologie linguistique dont nous n'allons pas rappeler la maturation - fonctionne donc comme cadre.restrictif à l'égard de la parenté postulée pour des états de langue observés: au XIXe siècle cette typologie est centrée sur la composition morphologique de mots, et non sur l'absence/présence de certaines classes de mots ni sur l'ordre des mots. Elle se définit par rapport au paramètre de comparaison choisi: la forme grammaticale. De plus, elle s'applique à des états de langue attestés: on n'y trouve pas de réflexion à propos de types possibles et impossibles (élément théorique devenu prominent dans

18 V'oir à ce propos notre article "Linguistique générale ê linguistique romane chez, Hugo Schuchardt", à paraître dans les Actes du XVIIle Congrès de Linguistique et de Philologie romanes (Trèves, 1986), éd. par D. Kremer.

19 A. Meillet, La méthode comparative en linguistique historique, Oslo - Paris, Aschehoug - Champion, 1925, p. 27.

20. Traduction de F. Schlegel. Über die Sprache und Weisheit der Indier. Ein Beitrag zur Begrundung der Altertumskunde. Heidelberg. Mohr \& Zimmer, 1808, p. 28. 
les recherches sur la reconstruction de la phonologie et de la syntaxe de l'indoeuropéen $)^{21}$.

La troisième composante de la grammaire comparée est la notion de "changements systématiques" en tant que transitions. La grammaire comparée ne peut ne pas être historique: il y a non seulement son objectif fondamental (de démontrer la parenté génétique de certaines langues), mais aussi sa matérialité première (des états de langue historiquement dispersés) qui la rendent historique. La notion de changement systématique comme transition correspond à un troisième problème de la comparaison: celui du non sequitur de la comparaison. En effet, l'aporie fondamentale de la comparaison - comme l'avait bien noté Meillet - est qu'on ne peut jamais démontrer que deux langues ne sont pas apparentées. Mais on a vu que la typologie permet déjà de renforcer la probabilité d'une hypothèse de parenté génétique; au niveau de l'explication des dissemblances, l'hypothèse gagnera en probabilité si l'on réussit à dégager des lois de transformation. Il est intéressant de remarquer que les lois établies par la grammaire comparée diffèrent sur un point essentiel des régularités reconnues par les linguistes perspicaces du XVIe et du XVIIe siècle, tels que Fernâo de Oliveira, Bernardo Aldrete ou le Père Besnier ${ }^{22}$ : la différence, c'est que les lois de la grammaire comparée ne concernent pas des mots juxtaposés (par ex. formes italiennes, espagnoles et portugaises; formes picardes, françaises et italiennes, etc.), mais qu'elles définissent des processus de transition par rapport à des séries (de phonèmes, en général). Il faut noter que cette composante théorique complète celle de la typologie linguistique: si la dernière définit le cadre (conditionnel) des concordances et dissimilitudes, la notion de changement phonétique en tant que transition permet de saisir l'actuation d'une différenciation à l'intérieur du type ${ }^{23}$ (on sait que chez Schleicher cette différenciation est située historiquement et spatialement). De cette façon, la troisième composante - correspondant à l'aporie fondamentale de la grammaire comparée - permet de montrer quelles évolutions sont plus probables, parce que plus systématiques.

${ }^{21}$ Cf. par exemple T. V. Gamkrelidze - V. V. Ivanov, "Sprachtypologie und die Rekonstruktion der gemeinindogermanischen Verschlïsse", Phonetica 27, 1973, pp. 150-156; idd., Indoevropejskij jazvk $i$ indoevropejcy, Tbilisi, Izd. Tbilisskogo Univ., 1984 (2 volumes); T. V. Gamkrelidze, "Language tvpology and language universals and their implications for the reconstruction of the IndoEuropean stop svstem", dans Y. Arbeitman - A. R. Bomhard éds. Bono homini donum. Essavs in historical linguistics, in memory of J. Alexander Kerns, Amsterdam, J. Benjamins, pp. 571-609; P. Ramat éd., Linguistic Reconstruction and Indo-European Svntax, Amsterdam, J. Benjamins, 1980; V. V. Shevoroshkin - T. L. Markey éds, Typology, Relationship, and Time, Ann Arbor, Karoma, 1986.

22 Pour les conceptions de ces auteurs, voir E. Coseriu, "Taal en functionaliteit' hei Fernão de Olivei$r a^{\prime}$, dans W. Abraham éd., Ut videam. Contributions to an understanding of linguistics, Lisse, Peter de Ridder, 1975, pp. 67-90; H.-M. Gauger, "Bernardo Aldrete (1565-1645). Ein Beitrag zur Vorgeschichte der romanischen Sprachgeschichte", Romanistisches Jahrbuch 18, 1967, pp. 207-248; et mes remarques à propos de P. Besnier, La réunion des langues, ou l'art de les apprendre toutes par une seule (éd. par V. Lo Cascio; Dordrecht, Foris, 1984) dans Bulletin de la Société de Linguistique de Paris 81: 2, 1986, pp. 74-79.

23 Voir à ce propos P. Swiggers, "Antoine Meillet et la méthode en linguistique", dans H. Aarsleff - L. G. Kelly - H.-.J. Niederehe, Papers in the Historv of Linguistics. Proceedings of the. Third International Conference on the History of the Language Sciences (ICHoLS III). Amsterdam, Benjamins 1987. pp. 595-606. 
Nous avons ainsi esquissé les contours conceptuels de la grammaire comparée, dans son rapport contrastif avec le comparatisme. Le modèle de la grammaire comparée ne s'est pas constitué d'emblée comme un système achevé; il manquait plusieurs chaînons dans la description de l'actuation des changements (influence de l'analogie et d'autres lois du langage - comme celles postulées par Bréal; l'importance des situations de bilinguisme (Ščerba/Meillet), les effets de l'apprentissage de langues par adultes et par enfants sur le développement des langues; les effets diachroniques de la norme $)^{24}$. Mais ce modèle a une individualité qui lui est propre et qui permet de le distinguer - par sa "technicité" — de la comparaison linguistique aux XVIIe et XVIIIe siècles. Il porte d'autre part l'empreinte de son objet: les langues indoeuropéennes, et dans une moindre mesure les langues sémitiques et les langues finno-ougriennes. Pour ces langues, la comparaison consiste à expliquer des mutations de segments dans des formes. On peut se demander quel aurait été l'aspect du modèle comparatiste si son objet avait été constitué par des langues telles que celles de la famille aranda, où les changements se font régulièrement sur le mode de la supplétion (conditionnée entre autres par des faits de tabou) ${ }^{25}$. Ces réflexions méthodologiques à propos de la comparaison de langues et de la pratique comparatiste débouchent ainsi sur un problème d'épistémologie historique des sciences du langage, qui montre les limites du savoir occidentảl, replié sur lui-même.

Povzetek

\section{OD PRIMERJAVE JEZIKOV DO PRIMERJALNE SLOVNICE METODOLOŠKA RAZMIŚLJANJA}

Avtor se posveča problemu, ki zadeva historiografijo in metodologijo jezikoslovja: prehodu od primerjanja jezikov do primerjalne slovnice. Zasnova primerjalne slovnice 19. stoletja se seveda naslanja na primerjavo jezikov, tako kot je že bilo od renesanse dalje, uvaja pa neko novost, značilno za novi ideal znanstvenosti. Ta nova perspektiva temelji na treh prvinah: na pojmu "slovnične oblike", na pojmu "jezikoslovnega modela" in na pojmu "sistemskih sprememb", dojetih kot prehodnih stanj. Vsaka od teh prvin se nanaša na neki vidik dela komparativistov (parametri, območje in metodološka omejenost jezikoslovnega primerjanja).

Model primerjalne slovnice, $k i$ je tako močno zaznamenovan s predmetom študija (indoevropski jeziki, v manjši meri semitski in ugro-finski jeziki) vsebuje tudi teoretske pomanjkljivosti, npr. ravno to, kar zadeva razlage jezikovnih sprememb.

24 Cf. M. Bréal, Essai de sémantique. Science des significations, Paris, Hachette, 1897; E. Haugen, The Ecology of Language. Essays selected and introduced by Anwar S. Dil, Stanford, Stanford University Press, 1972; U. Weinreich, Languages in Contact. Findings and problems, New York, Linguistic Circle, 1953; P. Swiggers, "La linguistique historique devant la variation: le cas de Meillet". Recherches sur le français parlé 7, 1985. pp. 61-74.

25. Voir à ce propos les remarques de N. Boretzky, "Das indogermanische Sprachwandelmodell und Wandel in exotischen Sprachen“, Zeitschrift für vergleichende Sprach forschung 95, 1982, pp. 49-80. 